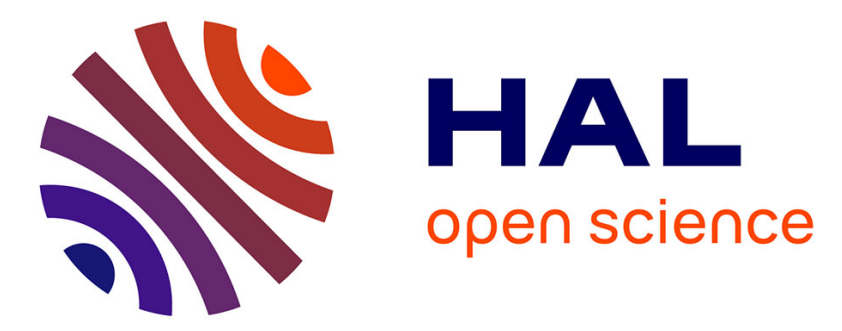

\title{
Genetic variability and transgenerational regulation of investment in sex in the monogonont rotifer Brachionus plicatilis
}

Océane Seudre, Eloïse Vanhoenacker, Stéphane Mauger, Jérôme Coudret, Denis Roze

\section{To cite this version:}

Océane Seudre, Eloïse Vanhoenacker, Stéphane Mauger, Jérôme Coudret, Denis Roze. Genetic variability and transgenerational regulation of investment in sex in the monogonont rotifer Brachionus plicatilis. Journal of Evolutionary Biology, inPress, 10.1111/jeb.13554 . hal-02407896

\section{HAL Id: hal-02407896 https://hal.science/hal-02407896}

Submitted on 12 Dec 2019

HAL is a multi-disciplinary open access archive for the deposit and dissemination of scientific research documents, whether they are published or not. The documents may come from teaching and research institutions in France or abroad, or from public or private research centers.
L'archive ouverte pluridisciplinaire HAL, est destinée au dépôt et à la diffusion de documents scientifiques de niveau recherche, publiés ou non, émanant des établissements d'enseignement et de recherche français ou étrangers, des laboratoires publics ou privés. 
Genetic variability and transgenerational regulation of investment in sex in the monogonont rotifer Brachionus plicatilis

Océane Seudre $^{1, *,}$, Eloïse Vanhoenacker ${ }^{1, *, \S}$, Stéphane Mauger ${ }^{*, \S}$, Jérôme Coudrett,§ and Denis Roze*,

* CNRS, UMI 3614, Evolutionary Biology and Ecology of Algae, Roscoff, France $\S$ Sorbonne Universités, UPMC Université Paris VI, Roscoff, France 1: equal contributions of both authors

Running title: Sex regulation in monogonont rotifers

Address for correspondence: Denis Roze, Station Biologique de Roscoff, Place Georges Teissier, CS90074, 29688 Roscoff Cedex, France Phone: (+33) 2564521 39, Fax: (+33) 298292324 
2 In cyclical parthenogens such as aphids, cladocerans and rotifers, the coupling

3 between sexual reproduction and the production of resting stages (diapausing eggs)

4 imposes strong constraints on the timing of sex. While induction of sex is generally

5 triggered by environmental cues, the response to such cues may vary across individuals

6 according to genetic and non-genetic factors. In this study, we explored genetic and

7 epigenetic causes of variation for the propensity for sex using a collection of strains from

8 a Spanish population of monogonont rotifers (Brachionus plicatilis) in which variation

9 for the threshold population density at which sex is induced (mixis threshold) had been

10 documented previously. Our results show significant variation for the mixis threshold

11 among 20 clones maintained under controlled conditions for 15 asexual generations.

12 The effect of the number of clonal generations since hatching of the diapausing egg

13 on the mixis ratio (proportion of sexual offspring produced) was tested on 4 clones

14 with contrasted mixis thresholds. The results show a negative correlation between the

15 mixis threshold and mixis ratio, as well as a significant effect of the number of clonal

16 generations since fertilization, sex being repressed during the first few generations after

17 hatching of the diapausing egg.

18

19 Keywords: cyclical parthenogenesis, monogonont rotifers, reproductive mode, sexual

20 reproduction, zooplankton 
22 Cyclical parthenogenesis (the alternation of phases of asexual propagation with sexual reproduction events) occurs in seven taxonomic groups of animals (Hebert, 1987), but has been best described in cladocerans, aphids and monogonont rotifers 25 (e.g., Simon et al., 2002; Wallace et al., 2006; Decaestacker et al., 2009). In all three taxa, individuals reproduce asexually while environmental conditions are favorable, 27 sex being often triggered by deteriorating conditions and leading to the production of diapausing eggs that can survive adverse conditions. This coupling between sexual reproduction and resting stage formation imposes a strong constraint on the timing of so sex (e.g., Gerber et al., 2018): sex should occur early enough to allow the production 31 of diapausing eggs before the environment becomes too hostile, but not too early 32 otherwise it will have an important cost in terms of reduced clonal propagation. The з3 timing of sex is thus tightly regulated in cyclical parthenogens, using different types 34 of environmental cues such as temperature (Simon et al., 2002), photoperiod (Zhang 35 and Baer, 2000), food quality (Lubzens et al., 1993; Koch et al., 2009) or crowding ${ }_{36}$ (Stelzer and Snell, 2003; Gerber et al., 2018). Sensitivity to those cues may vary 37 among populations and reflect local adaptation to different environmental conditions 38 (e.g., Roulin et al., 2013; Franch-Gras et al., 2017); whether genetic variation also exist 40 Agrawal, 2010).

${ }_{41} \quad$ Facultatively sexual organisms are of particular interest for designing experi42 mental tests of the evolutionary significance of sex and recombination. While many of 43 these tests compared the rates of adaptation of sexual vs. asexual populations of uni- 
44 cellular organisms (e.g., Kaltz and Bell, 2002; Colegrave, 2002; Goddard et al., 2005;

45 Lachapelle and Bell, 2012; McDonald et al., 2016), several experiments on brachionid 46 rotifers took advantage of the existence of variability in investment in sex among indi47 viduals, in order to explore under which conditions increased (or decreased) investment 48 in sex may be favored (e.g., Smith and Snell, 2006; Becks and Agrawal, 2010, 2012;

Luijckx et al., 2017). While these studies have lead to important insights, different components of investment in sex may vary between individuals (threshold level of the cue triggering sex, proportion of sexual offspring produced once the threshold has been reached); furthermore, this variation may be genetic or epigenetic, with some non-genetic effects possibly lasting over several generations (Gilbert, 2017). The aim of the present paper is to provide a better understanding of these different sources of variation (and how they may covary), which appears of particular importance for the design and interpretation of evolution experiments.

Monogonont rotifers are small invertebrates (50 to $2000 \mu \mathrm{m}$ ) living in a variety of aquatic or moist habitats (Wallace et al., 2006), often reaching very large population sizes due to high rates of clonal reproduction. Rotifer populations are typically temporary at temperate latitudes, the growing season starting by the hatching of sexually produced diapausing eggs present in the sediment. The hatchlings are diploid asexual (amictic) females, producing other females by ameiotic parthenogenesis. After an initial phase of population growth, sexual and asexual reproduction co-occur within populations: sex is induced by an environmental factor, causing parthenogenetic females to produce some sexual (mictic) females among their offspring (see Figure 1). These sexual females produce haploid eggs by meiosis, which, if not fertilized, develop into dwarf haploid males. If sexual females are inseminated while they are still young, 
72 become favorable again.

they produce diploid diapausing eggs formed by regular gamete fusion. These diapausing "eggs" actually consist in multicellular embryos that can resist dessication and adverse environmental conditions, and may remain viable for several years (Lubzens et al., 2001). After a dormant phase, they can hatch when the environmental conditions In rotifers from the Brachionus genus, the switch from asexual to sexual reproduction is mainly controlled by population density, through a form of quorum sensing mechanism involving a protein (the "mixis-inducing protein" or MIP) produced by the females themselves (Carmona et al., 1993; Stelzer and Snell, 2003, 2006; Snell et al., 2006). The threshold population density at which sexual females start being produced (called the "mixis threshold") was shown to vary among species, and also among strains from the same species (Gilbert, 2017 and references therein). Variations in the mixis threshold among isolates from the same natural population was demonstrated in the brackish-water species Brachionus plicatilis (Carmona et al., 2009; Gabaldon and Carmona, 2015), and in the freshwater species Brachionus calyciflorus (Becks and Agrawal, 2010). A second component of investment in sex is the "mixis ratio", corresponding to the proportion of sexual females among offspring (once the mixis threshold has been reached — see Figure 1); variation for the mixis ratio among strains has also been demonstrated, but to what extent the mixis ratio correlates with the mixis threshold remains unclear (Gilbert, 2017).

Other environmental factors such as salinity or food quality may also affect investment in sex in monogonont rotifers (e.g., Lubzens et al., 1993). Interestingly, Gilbert $(2002,2003)$ showed that in B. calyciflorus, the mixis ratio is affected by endogenous factors that may persist over several generations: in particular, sexual re- 
production is inhibited during the first clonal generations following fertilization, with a gradual increase in the mixis ratio over the first 10-12 clonal generations after hatching of a diapausing egg. The same pattern was observed in different monogonont species, but was absent in others (e.g., Schröder and Gilbert, 2004; Gilbert, 2017). Although Gilbert (2003) and Schröder and Gilbert (2004) observed variation between genotypes from the same natural population in the rate of increase of the mixis ratio over clonal generations, the heritable component of this variation cannot be assessed from these experiments, since only a single replicate per genotype was performed. Conversely, this type of transgenerational effect may possibly have affected previous estimates of genetic variation for the mixis threshold, as the number of clonal generations since hatching of the diapausing egg is generally not strictly controlled in the experiments in which this variation is measured.

In this study, we first quantified genetic variation for the mixis threshold among a collection of strains originating from the same natural population of Brachionus plicatilis, measured after 15 clonal generations under controlled conditions. In a second experiment, we used a subset of 4 strains with contrasted mixis thresholds, to assess the effect of the number of clonal generations after fertilization on the mixis ratio. This allowed us to asses how these two measures of investment in sex (mixis threshold and mixis ratio) may covary, as well as to test the existence of possible transgenerational effects on induction of sex that have been described in other species. The results from our second experiment also allowed us to test for the effect of another non-genetic factor, the age of the mother, on the proportion of sexual offspring produced. 
Isolation and culture conditions of rotifer strains. Clones of B. plicatilis were obtained from the hatching of diapausing eggs present in sediment sampled from Salobralejo Lake (Eastern Spain) in September 2013, and kindly provided by the Laboratory of Evolutionary Ecology of the University of Valencia. This particular population was chosen because genetic variation for the mixis threshold had been documented in previous studies (Gabaldon and Carmona, 2015; Franch-Gras et al., 2017). Diapausing eggs were extracted from the sediment using the sugar flotation technique (Gómez and Carvalho, 2000), and hatched by placing them in artificial seawater (Instant Ocean ${ }^{\circledR}$, Aquarium Systems) at $6 \mathrm{~g} / \mathrm{L}$ salinity, under constant illumination and at $22^{\circ} \mathrm{C}$. Upon hatching, individuals were transferred to culture medium (referred hereafter as standard culture medium) consisting in f/2-enriched artificial seawater (Guillard, 1975) at $12 \mathrm{~g} / \mathrm{L}$ salinity, containing $2 \times 10^{5}$ cells $/ \mathrm{mL}$ of the microalga Tetraselmis suecica used for food (our algal culture was maintained in exponential growth in a chemostat throughout the experiment). Because two cryptic species of rotifers (B. plicatilis and B. manjavacas) coexist in Salobralejo Lake (Montero-Pau et al., 2011), the first offspring of each hatched individual was collected for species identification using the RFLP-PCR method described in Gabaldon et al. (2013) — in parallel, we devised a quicker identification method using COI DNA primers (described in the Supplementary Material) that yielded identical results. Twenty hatched B. plicatilis individuals were identified, and individually transferred into $30 \mathrm{~mL}$ glass tubes containing standard culture medium to maintain clonal growth. Clones were then maintained by weekly transfers to fresh medium. Hereafter, these 20 clones will be denoted "P-clones" (for 
Variability in the mixis threshold among clones. Three asexual females from each of the 20 P-clones were sampled and individually transferred into wells of 48-well culture plates (Greiner Bio-One ${ }^{\mathrm{TM}}$ ) containing $0.3 \mathrm{~mL}$ of standard culture medium and maintained in a culture chamber at $22^{\circ} \mathrm{C}$, in order to generate sub-clonal lines $(3$ per P-clone). Their first offspring were transferred into new wells with fresh medium, until 15 clonal generations were reached (when a sampled offspring was a sexual female, it was replaced by another offspring from the same mother until obtaining an asexual female). This high number of clonal generations was chosen to ensure that none of the tested females could be just a few generations away from hatching of a diapausing egg, given that diapausing eggs may hatch spontaneously under culture conditions (e.g., Martínez-Ruiz and García-Roger, 2015). For each sub-clonal line, 6 neonates of the fifteenth generation were isolated and individually transferred into wells of 24-well culture plates (Greiner Bio-One ${ }^{\mathrm{TM}}$ ) containing $0.5 \mathrm{~mL}$ of culture medium with an algal concentration of $5 \times 10^{5}$ cells $/ \mathrm{mL}$, where they were let to reproduce. If the sampled female was sexual, it was replaced whenever possible by another fifteenth generation female from the same mother. Wells were inspected visually every $24 \mathrm{~h}$ until the first males were observed, in which case the population density was measured by counting the number of females present in the well. This density corresponds to the estimated mixis threshold (Carmona et al., 2009).

Effect of the number of clonal generations after diapausing egg hatching. A subset of $4 \mathrm{P}$-clones displaying constrasted mixis thresholds was chosen based on 
the results of the previous experiment (clones 6, 8, 10 and 16, see Results section). Diapausing eggs were collected from the bottom of the tubes in which those clones were maintained, transferred into Petri dishes containing artificial seawater at $12 \mathrm{~g} / \mathrm{L}$ salinity, and maintained in the dark and at $4^{\circ} \mathrm{C}$ during 3 months. These diapausing eggs were produced by intraclonal mating, which is genetically equivalent to selffertilization in hermaphroditic organisms. Diapausing eggs were then isolated into single wells of 48 -well plates (Greiner Bio-One ${ }^{\mathrm{TM}}$ ) with $0.3 \mathrm{~mL}$ of standard medium, and placed at $22^{\circ} \mathrm{C}$ and under constant illumination to induce hatching. For each P-clone, five hatched females were sampled at random to form our first generation (G1, see Supplementary Figure). Note that these five females have been produced by different intraclonal fertilization events, and thus carry different genotypes (however, two females from the same P-clone are more related than females from two different P-clones). The first three juveniles (G2) produced by each G1 female were collected to initiate sub-clonal lines. Hereafter, we will denote "F1-clone" the set of 3 sub-clonal lines originating from the same G1 female (5 per P-clone); note that all individuals from the same F1-clone are genetically identical, since they are produced asexually. Sub-clonal lines were maintained in 48-well plates (Greiner Bio-One ${ }^{\mathrm{TM}}$ ) that were inspected daily. When a female of a given generation had produced its first juvenile, the juvenile was transferred into a new well with $0.3 \mathrm{~mL}$ of fresh standard culture medium. If the juvenile developed into a sexual female or died before reproducing, it was replaced by another juvenile produced by the same mother. Sub-clonal lines were maintained until the 24th generation (G24), at the exception of sub-clonal lines from clone 8 , which took more time as more sexual females were produced, and which were maintained for 18 generations only. At generations 2, 5, 8, 12, 18 and 24, one 
juvenile female was sampled from each sub-clonal line to measure its mixis ratio. For this, the tested female was placed in a well of a 48-well plate (Greiner Bio-One ${ }^{\mathrm{TM}}$ ) with $0.3 \mathrm{~mL}$ of sex-inducing medium, consisting in standard culture medium with an algal concentration of $4 \times 10^{5}$ cells $/ \mathrm{mL}$, mixed in equal proportions with filtrate obtained from a previous rotifer culture that had reached a density of approximately 20 females $/ \mathrm{mL}$ (filtrated on a $0.2 \mu \mathrm{m}$ mesh), and that was stored at at $5^{\circ} \mathrm{C}$ (a unique batch of this medium was used throughout the experiment). The concentration of mixis-inducing protein in the resulting medium should thus be approximately equivalent to its concentration in a population at 10 females/mL density, which is well above the density required to induce sex in most populations (Gilbert, 2017). Every day until its death (which generally occurred after 10 to 15 days), the tested female was transferred into a new well containing $0.3 \mathrm{~mL}$ of fresh sex-inducing medium, and its offspring were collected and individually transferred to a single well of a 96-well plate (Greiner Bio-One ${ }^{\mathrm{TM}}$ ) containing $0.2 \mathrm{~mL}$ of standard culture medium. When offspring started to reproduce, they were typed as asexual (if they produced females) or sexual (if they produced males).

Data analyses. The mixis threshold (measured in number of females per mL at the time of first male appearance) in the first experiment was log-transformed and analyzed by fitting a mixed effects linear model, with 'P-clone' as a fixed effect (with 20 levels corresponding to the different P-clones) and 'sub-clonal line' (three for each P-clone) as a random effect. Mixis ratios in the second experiment were analyzed using a generalized linear mixed effects model (GLMM), in which the numbers of sexual/asexual females produced per day by each tested female was modelled as a bi- 
nomial variable with a logit link function. The model included 'P-clone' (with 4 levels), 'tested generation' (number of clonal generations from the diapausing egg, treated as a continuous variable), 'day of the reproductive period' (day 1 corresponding to the first day the tested female produced a juvenile, treated a a continuous variable) as fixed effects, as well as an interaction between 'P-clone and 'tested generation', and an interaction between 'P-clone' and 'day of reproductive period'. Effects of the F1-clone ( 5 for each P-clone) and of the sub-clonal line (3 for each F1-clone) were included in the model as random effects. The correlation between the mixis threshold (measured in the first experiment) and mixis ratio (measured in the second experiment) over the $4 \mathrm{P}$-clones used in the second experiment was assessed using a modified version of the model for the second experiment, in which 'tested generation' and 'mixis threshold' (one estimate for each P-clone) were included as fixed effects, while 'F1-clone' and 'subclonal line' were included as random effects. For this last analysis, we only included mixis ratios measured at generations 8,12 and 18. The significance of fixed effects and their interactions was assessed by comparing models with or without the tested effect or interaction using likelihood ratio tests. Analyses were carried out using R v. 3.3.3 (R Core Team, 2017), and the lmer, glmer and anova functions from the "Ime4" package (Bates et al., 2015). Marginal coefficients of determination (proportions of the variance explained by fixed effects) were obtained using the r.squaredGLMM function 8 of the "MuMIn" package (Barton, 2018). 
242

Variability in mixis threshold. Figure 2 shows the average density at first male appearance for the different P-clones, measured after 15 asexual generations. As explained in the Methods, three sub-clonal lines were maintained for each of the P-clones, and 6 measures were performed for each sub-clonal line (yielding 18 measures per Pclone). However, some sub-clonal lines were lost (due to the death of individuals that could not be replaced), and as a consequence, we obtained results from only two subclonal lines (instead of three) from P-clones 1, 2 and 15. The number of measures from some of the other sub-clonal lines was less than 6 due to the early death of individuals, yielding a total of 326 mixis threshold estimates (instead of 360). The statistical analysis showed significant variation among P-clones for their mixis thresholds $\left(\chi^{2}(19)=36.17, p=0.01\right)$. The proportion of total variance explained by the 'P-clone' effect was 0.16 .

Transgenerational effect on the mixis ratio. From the previous results, we selected two P-clones with high and low estimated mixis thresholds (clones 6 and 8, respectively - see Figure 2), and two P-clones with near-average mixis thresholds (clones 10 and 16) to perform the experiment on the effect of the number of clonal generations after fertilization on investment in sex. A total of 15 sub-clonal lines were maintained for each of these P-clones (see Material and Methods, Supplementary Figure), starting from 5 diapausing eggs produced by intraclonal mating ( 3 sub-clonal lines per diapausing egg). However, the lines originating from one of the diapausing eggs from clone 10 had low fitness (high death rates of individuals and low fecundity, which 
may be caused by inbreeding depression) and these lines could not be maintained: therefore, data from clone 10 consist in measurements over 4 distinct genotypes (F1clones) produced by intraclonal mating (instead of 5). Furthermore, one data point was missing for clone 8 at generation 5, and two at generation 18 (leading to 14 and 13 measures instead of 15$)$, due to the premature death of tested females.

Figure 3 shows the effect of the number of generations since hatching of the diapausing egg on the mixis ratio (measured as the proportion of sexual females produced among all offspring produced by a female), averaged over each P-clone (results for all F1-clones are shown in Figure 4). The results show a significant increase of the mixis ratio with the number of clonal generations $\left(\chi^{2}(4)=241.60, p<0.001\right)$, G2 individuals (that is, the offspring of individuals that hatched from diapausing eggs) producing very few sexual females, while the mixis ratio increases to reach a plateau after about 8 to 10 clonal generations. The results also show significant differences among P-clones $\left(\chi^{2}(9)=910.29, p<0.001\right)$, with a much higher mixis ratio of individuals from clone 8 (observed over all 5 F1-clones, see Figure 4), while the mixis ratio is lowest in individuals from clone 6 . The model also detected a significant interaction between the tested generation and P-clone $\left(\chi^{2}(3)=41.64, p<0.001\right)$, reflecting the fact that the mixis ratio increases more rapidly with the number of clonal generations in some P-clones than others. The model in which different coefficients were attributed to the four P-clones was significantly better than a model in which clones 6 and 16 were treated as identical $\left(\chi^{2}(3)=15.53, p=0.0014\right)$, and was also better than a model in which clones 10 and 16 were treated as identical $\left(\chi^{2}(3)=28.89, p<0.001\right)$, reflecting the fact that the four P-clones displayed different behaviors. Finally, our modified statistical model including the estimated mixis threshold of P-clones as a fixed factor (see 
Material and Methods) detected a significant, negative effect of the mixis threshold on the mixis ratio $\left(\chi^{2}(1)=504.61, p<0.001\right)$, indicating that $\mathrm{P}$-clones with lower mixis thresholds tend to have higher mixis ratios.

Effect of maternal age on the mixis ratio. The model detected a significant effect of the age of the tested mother ('day of the reproductive period' effect) on the proportion of sexual females produced per day $\left(\chi^{2}(4)=177.54, p<0.001\right)$, and a significant interaction between 'P-clone' and 'day of the reproductive period' $\left(\chi^{2}(3)=14.70\right.$, $p=0.002$ ). Indeed, Figure 5 shows that the tested females tended to produce a higher proportion of sexual offspring during their first days of reproduction, the decline in mixis ratio with the age of the mother being most apparent for clone 8 .

\section{DISCUSSION}

Although one may expect that the timing of sex should be under strong selection in cyclical parthenogens, substantial genetic variation in the rate of response to the sex-inducing stimulus may exist within natural populations (e.g., Carmona et al., 2009; Becks and Agrawal, 2010). Our results confirm the existence of genetic variability for the threshold population density to induce sex within a single population of the monogonont rotifer Brachionus plicatilis, after controlling for the number of clonal generations since fertilization (at least 15 in our first experiment). Our estimate for the proportion of variance in the mixis threshold explained by the genotype of individuals (0.16) is lower than the heritability estimates obtained by Gabaldon and Carmona (2015) and Franch-Gras et al. (2017) from the same natural population 
(0.51 and 0.36, respectively). This difference may partly be due to the fact that the number of clonal generations since hatching of the last diapausing egg was not strictly controlled in these previous experiments (although three clonal generations were performed before the estimation of density thresholds). Perhaps more likely, it may be caused by a higher environmental variance in our experiment. In particular, males were observed at densities much higher than in Gabaldon and Carmona's experiment, which may stem from the fact that density thresholds were assessed in smaller volumes in our experiment $(0.5 \mathrm{~mL}$, vs. $15 \mathrm{~mL}$ in Gabaldon and Carmona, 2015, Franch-Gras et al., 2017). Indeed, the mixis threshold estimate is known to be negatively correlated to the culture volume, which may be due to the fact that in smaller volumes, population density may reach higher values before the mixis-inducing protein reaches the concentration needed to induce sex (Carmona et al., 2011). A higher number of reproductive events during the time needed to reach the mixis threshold may possibly have enhanced the effect of environmental factors on the estimated density at the threshold. Furthermore, our test populations were observed once per $24 \mathrm{~h}$ (vs. twice in Gabaldon and Carmona's study), which may also have inflated the variance caused by measurement error.

Our second experiment showed important genetic variation in the mixis ratio (proportion of sexual offspring produced under a strong sex-inducing stimulus), and a correlation between the two components of propensity for sex: the clone in which the density at first male appearance was the lowest (respectively, highest) in the first experiment displayed the highest (respectively, lowest) mixis ratio in the second experiment (clones 6 and 8, Figures 1 and 2). This result shows that genotypes do indeed differ 1 in their overall investment in sexual reproduction; a different conclusion would have 
been reached if the genotypes engaging early in sexual reproduction also had tended to show a lower investment in sex under a strong sex stimulus (that is, if the correlation between mixis threshold and mixis ratio had been positive). One may notice that our sex-inducing medium was equivalent to a density of 10 individuals $/ \mathrm{mL}$, which is much lower than the mixis thresholds shown in Figure 1. However, this discrepancy again stems from the fact that when estimating mixis thresholds by the density at first male appearance in growing populations, the measured density is likely to be much higher than the density that would be required to produce the threshold concentration of mixis-inducing protein in a steady-state population, particularly when measurements are performed in small volumes (Carmona et al., 2011). In order to test whether a higher density would increase the mixis ratio, we ran additional tests on 10 females from clone 6, after 18 generations from the diapausing egg, exposing them to a sexinducing medium corresponding to 25 individuals/mL (instead of 10), but we did not observed any significant increase of the mixis ratio (results not shown). Our results also show that young asexual females tend to produce a higher proportion of sexual offspring than older females, in agreement with previous observations on B. plicatilis (Carmona et al., 1994), B. calyciflorus (Rougier and Pourriot, 1977) and Synchaeta tremula (Timmermeyer and Stelzer, 2006) - however, a maximal investment in sex in the middle of the reproductive period of individuals was observed in one study on B. calyciflorus (Fussmann et al., 2007).

The selective forces allowing the maintenance of genetic polymorphism for investment in sex within natural populations remain unknown. Carmona et al. (2009) showed that clones investing less in sex tend to increase in frequency during the growing season (since they invest more in parthenogenetic reproduction), and hypothesized 
that temporal fluctuations in the length of growing seasons may allow the maintenance of polymorphism, as genotypes investing more in the production of diapausing eggs may be favored when growing seasons are short, while genotypes investing more in parthenogenetic growth may be favored under longer growing seasons (see also Franch-Gras et al., 2017). Theoretical models have shown that temporal environmental fluctuations coupled with a dormant stage can indeed allow the maintenance of polymorphism (the "storage effect", e.g., Warner and Chesson, 1985; Ellner and Hairston, 1994; Turelli et al., 2001), and a model by Spencer et al. (2001) showed that reproductive strategies differing in the timing of diapausing egg production may coexist within the same population when the time length of the growing season is uncertain.

Finally, our results demonstrate a gradual increase in the propensity for sex over clonal generations following the hatching of diapausing eggs. This confirms previous indications that a transgenerational maternal effect repressing sexual reproduction, and similar to the one observed in several other monogonont species (Gilbert, 2002; Schröder and Gilbert, 2004) occurs in B. plicatilis (Hino and Hirano, 1977; Hagiwara et al., 2005). This delayed-mixis mechanism may have evolved to increase the chances of establishment of newly hatched lineages, by promoting parthenogenetic growth (Serra et al., 2005) — while an increasing proportion of sexual daughters produced over the lifetime of the mother could also have evolved in order to postpone sexual reproduction, our results demonstrate that this is not the case, since young mothers tend to produce higher frequencies of sexual offspring (Figure 5). Several hypotheses have been proposed concerning the mechanism underlying this transgenerational effect (DNA methylation, cytoplasmic compound present in decreasing concentration over 
381

generations, e.g., Gilbert, 2017), but it currently remains unknown. While similar sex-repressing mechanisms may be favoured in other cyclical parthenogens (such as aphids or cladocerans) at the start of the growing season, their existence has (to our knowledge) not been tested. Interestingly, the existence of such a mechanism in monogonont rotifers raises the possibility that the effect of other factors known to affect investment in sex, such as population density or food stress, may persist over a given number of clonal generations (evidence that food stress may affect the mictic response of females over several generations can be found in Hagiwara et al., 2005; Kamizono et al., 2017). These effects should be explored in order to better understand the selective forces that may act on the evolution of the propensity for sex in monogonont rotifers, in both natural and experimental environments.

Acknowledgements. We thank María José Carmona, Eduardo García Roger and Eva Tarazona for providing rotifer strains, technical advice and useful discussion and comments on this manuscript, and Thomas Broquet, Christoph Haag, Thomas Lenormand, Manuela López Villavicencio, Henrique Teotónio and one anonymous reviewer for useful suggestions and comments. This work was supported by the French Agence Nationale de la Recherche (project SexChange, ANR-14-CE02-0001). 
Bartoń, K. 2018. MuMIn: multi-model inference. R package version 1.40.4, https://CRAN.R-project.org/package=MuMIn.

Bates, D., M. Maechler, B. Bolker, and S. Walker. 2015. Fitting linear mixed-effects models using lme4. J. Stat. Software 67:1-48.

Becks, L. and A. F. Agrawal. 2010. Higher rates of sex evolve in spatially heterogeneous environments. Nature 468:89-93.

- 2012. The evolution of sex is favoured during adaptation to new environments. PLoS Biology 5:e1001317.

Carmona, M. J., N. Dimas-Flores, E. M. García-Roger, and M. Serra. 2009. Selection for low investment in sex in a cyclically parthenogenetic rotifer. J. Evol. Biol. 22:1975-1983.

Carmona, M. J., N. Dimas-Flores, J. Montero-Pau, and M. Serra. 2011. Effect of experimental methodology on estimation of density at sex initiation in cyclically parthenogenetic rotifers. Hydrobiologia 662:131-139.

Carmona, M. J., M. Serra, and M. R. Miracle. 1993. Relationships between mixis in Brachionus plicatilis and preconditioning of culture medium by crowding. Hydrobiologia 255/256:145-152.

- 1994. Effect of population density and genotype on life-history traits in the rotifer Brachionus plicatilis O.F. Müller. J. Exp. Mar. Biol. Ecol. 182:223-235. 
Colegrave, N. 2002. Sex releases the speed limit on evolution. Nature 420:664-666.

Decaestacker, E., L. De Meester, and J. Mergeay. 2009. Cyclical parthenogenesis in Daphnia: sexual versus asexual reproduction. Pp. 295-316 in I. Schön, K. Martens, and P. van Dijk, eds. Lost sex, the evolutionary biology of parthenogenesis. SpringerVerlag, Dordrecht.

Ellner, S. and R. G. Hairston. 1994. Role of overlapping generations in maintaining genetic variation in a fluctuating environment. Am. Nat. 143:403-417.

Franch-Gras, L., E. García-Roger, M. Serra, and M. J. Carmona. 2017. Adaptation in response to environmental unpredictability. Proc. Roy. Soc. (Lond.) B 284:20170427.

Fussmann, G. F., G. Kramer, and M. Labib. 2007. Incomplete induction of mixis in Brachionus calyciflorus: patterns of reproduction at the individual level. Hydrobiologia 593:111-119.

Gabaldon, C. and M. J. Carmona. 2015. Allocation patterns in modes of reproduction in two facultatively sexual cryptic rotifer species. J. Plankton Res. 37:429-440.

Gabaldon, C., J. Montero-Pau, M. Serra, and M. J. Carmona. 2013. Morphological similarity and ecological overlap in two rotifer species. PLoS One 8:e57087.

Gerber, N., H. Kokko, D. Ebert, and I. Booksmythe. 2018. Daphnia invest in sexual reproduction when its relative costs are reduced. Proc. Roy. Soc. (Lond.) B 285:20172176.

Gilbert, J. J. 2002. Endogenous regulation of environmentally induced sexuality in a rotifer: a multigenerational parental effect induced by fertilization. Freshwater Biol. $47: 1633-1641$. 
- 2003. Environmental and endogenous control of sexuality in a rotifer life cycle: developmental and population biology. Evol. Dev. 5:19-24.

- 2017. Non-genetic polymorphisms in rotifers: environmental and endogenous controls, development, and features for predictable or unpredictable environments. Biol. Rev. 92:964-992.

Goddard, M. R., H. C. J. Godfray, and A. Burt. 2005. Sex increases the efficacy of natural selection in experimental yeast populations. Nature 434:636-640.

Gómez, A. and G. R. Carvalho. 2000. Sex, parthenogenesis and genetic structure of rotifers: microsatellite analysis of contemporary and resting egg bank populations. Mol. Ecol. 9:203-214.

Guillard, R. R. L. 1975. Culture of phytoplankton for feeding marine invertebrates. Pp. 29-60 in W. L. Smith and M. H. Chanley, eds. Culture of marine invertebrate animals. Springer, Boston, MA.

Hagiwara, A., Y. Kadota, and A. Hino. 2005. Maternal effect by stem females in Brachionus plicatilis: effect of starvation on mixis induction in offspring. Hydrobiologia $546: 275-279$.

Hebert, P. D. N. 1987. Genotypic characteristics of cyclic parthenogens and their obligately asexual derivatives. Pp. 175-195 in S. J. Stearns, ed. The evolution of sex and its consequences. Birkhäuser Verlag, Basel.

Hino, A. and R. Hirano. 1977. Ecological studies on the mechanism of bisexual reproduction in the rotifer Brachionus plicatilis II - Effect of cumulative parthenogenetic 
generation on the frequency of bisexual reproduction. Bull. Jap. Soc. Sci. Fish. 43:1147-1155.

Kaltz, O. and G. Bell. 2002. The ecology and genetics of fitness in Chlamydomonas. XII. Repeated sexual episodes increase rates of adaptation to novel environments. Evolution 56:1743-1753.

Kamizono, S., E. O. Ogello, Y. Sakakura, and A. Hagiwara. 2017. Effect of starvation and accumulation of generations on mixis induction in offspring of the monogonont rotifer Brachionus manjavacas hatched from resting eggs. Hydrobiologia 796:93-97.

Koch, U., E. von Elert, and D. Straile. 2009. Food quality triggers the reproductive mode in the cyclical parthenogen Daphnia (Cladocera). Oecologia 159:317-324.

Lachapelle, J. and G. Bell. 2012. Evolutionary rescue of sexual and asexual populations in a deteriorating environment. Evolution 66:3508-3518.

Lubzens, E., Y. Wax, G. Minkoff, and F. Adler. 1993. A model evaluating the contribution of environmental factors to the production of resting eggs in the rotifer Brachionus plicatilis. Hydrobiologia 255/256:127-138.

Lubzens, E., O. Zmora, and Y. Barr. 2001. Biotechnology and aquaculture of rotifers. Hydrobiologia 446/447:337-353.

Luijckx, P., E. K. H. Ho, M. Gasim, S. Chen, A. Stanic, C. Yanchus, Y. S. Kim, and A. F. Agrawal. 2017. Higher rates of sex evolve during adaptation to more complex environments. Proc. Natl. Acad. Sci. U. S. A. 114:534-539.

Martínez-Ruiz, C. and E. M. García-Roger. 2015. Being first increases the probability of long diapause in rotifer resting eggs. Hydrobiologia 745:111-121. 
McDonald, M. J., D. P. Rice, and M. M. Desai. 2016. Sex speeds adaptation by altering the dynamics of molecular evolution. Nature 531:233-236.

Montero-Pau, J., E. Ramos-Rodríguez, M. Serra, and A. Gómez. 2011. Long-term coexistence of rotifer cryptic species. PLoS One 6:e21530.

R Core Team. 2017. R: A Language and Environment for Statistical Computing. R Foundation for Statistical Computing, Vienna, Austria. https://www.Rproject.org/.

Rougier, C. and R. Pourriot. 1977. Aging and control of the reproduction in Brachionus calyciflorus (Pallas) (Rotatoria). Exp. Geront. 12:137-151.

Roulin, A. C., J. Routtu, M. D. Hall, T. Janicke, I. Colson, C. R. Haag, and D. Ebert. 2013. Local adaptation of sex induction in a facultative sexual crustacean: insights from QTL mapping and natural populations of Daphnia magna. Mol. Ecol. 22:35673579.

Schröder, T. and J. J. Gilbert. 2004. Transgenerational plasticity for sexual reproduction and diapause in the life cycle of monogonont rotifers: intraclonal, intraspecific and interspecific variation in the response to crowding. Funct. Ecol. 18:458-466.

Serra, M., T. W. Snell, and J. J. Gilbert. 2005. Delayed mixis in rotifers: an adaptive response to the effects of density-dependent sex on population growth. J. Plankton Res. 27:37-45.

Simon, J.-C., C. Rispe, and P. Sunnucks. 2002. Ecology and evolution of sex in aphids. Trends Ecol. Evol. 17:34-39. 
Smith, H. A. and T. W. Snell. 2006. Rapid evolution of sex frequency and dormancy as hydroperiod adaptations. J. Evol. Biol. 25:2501-2510.

Snell, T. W., J. Kubanek, W. Carter, A. B. Payne, J. Kim, M. Hicks, and C.-P. Stelzer. 2006. A protein triggers sexual reproduction in Brachionus plicatilis. Mar. Biol. 149:763-773.

Spencer, M., N. Colegrave, and S. S. Schwartz. 2001. Hatching fraction and timing of resting stage production in seasonal environments: effects of density dependence and uncertain season length. J. Evol. Biol. 14:357-367.

Stelzer, C. P. and T. W. Snell. 2003. Induction of sexual reproduction in Brachionus plicatilis (Monogononta, Rotifera) by a density-dependent chemical cue. Limnol. Oceanogr. 48:939-943.

- 2006. Specificity of the crowding response in the Brachionus plicatilis species complex. Limnol. Oceanogr. 51:125-130.

Timmermeyer, N. and C.-P. Stelzer. 2006. Chemical induction of mixis in the rotifer Synchaeta tremula. J. Plankton Res. 28:1233-1239.

Turelli, M., D. W. Schemske, and P. Bierzychudek. 2001. Stable two-allele polymorphisms maintained by fluctuating fitnesses and seed banks: protecting the blues in Linanthus parryae. Evolution 55:1283-1298.

Wallace, R. L., T. W. Snell, C. Ricci, and T. Nogrady. 2006. Rotifera 1: Biology, Ecology and Systematics. Backhuys Publishers, Leiden.

4 Warner, R. R. and P. L. Chesson. 1985. Coexistence mediated by recruitment fluctuations: a field guide to the storage effect. Am. Nat. 125:769-787. 
516 Zhang, L. and K. N. Baer. 2000. The influence of feeding, photoperiod and selected 517 solvents on the reproductive strategies of the water flea, Daphnia magna. Environ. 518 Pollut. 110:425-430. 


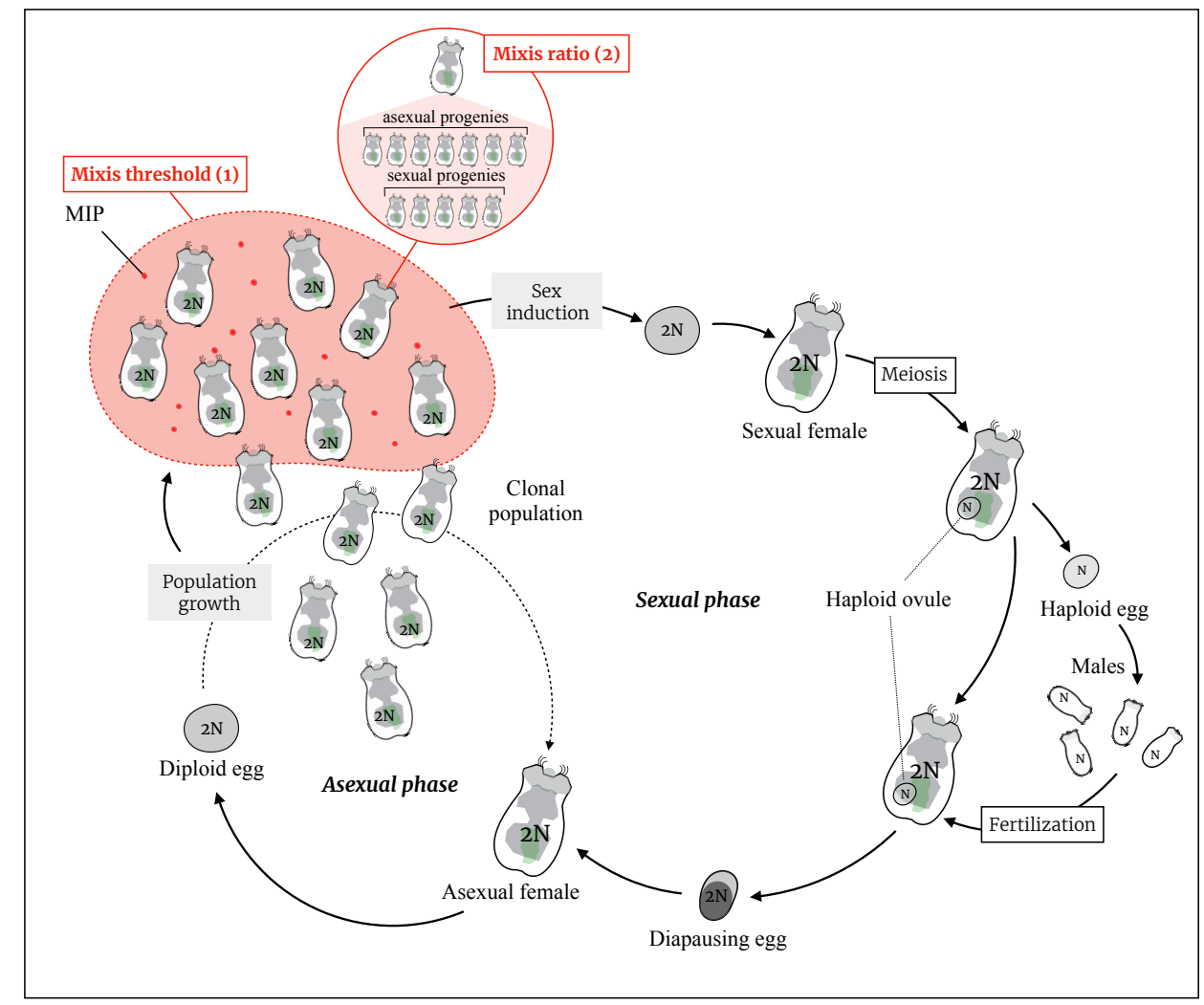

Figure 1. Brachionus plicatilis life cycle. Asexual reproduction leads to clonal pop-

${ }_{527}$ given female is referred to as its mixis ratio (2). 


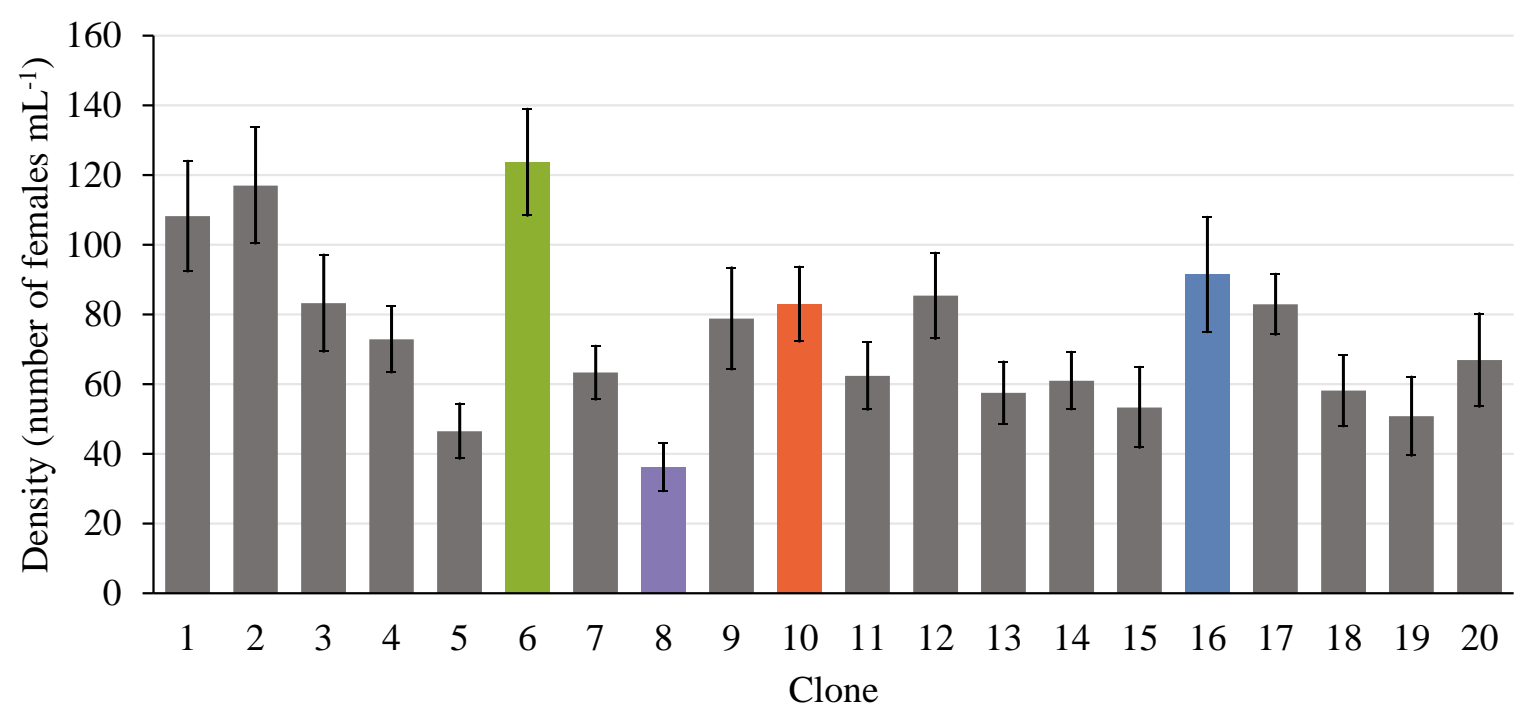

528

Figure 2. Average mixis threshold of the different P-clones (density at which the 530 first males were observed), measured after 15 asexual generations. Error bars show ${ }_{531} \pm 1$ S.E. The colored bars show the clones selected for the second experiment (effect of 532 the number of generations after fertilization on the mixis ratio). 


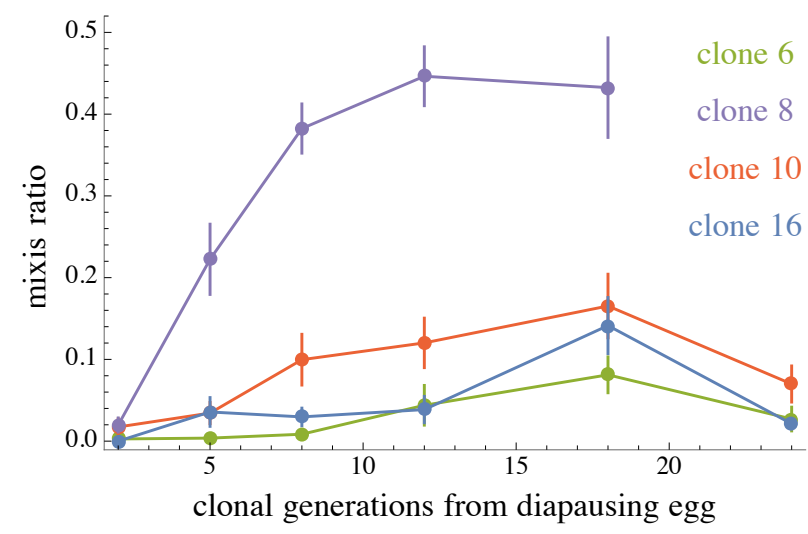

533

534 Figure 3. Mixis ratio (proportion of sexual females among the whole progeny of 535 an individual) averaged over each $\mathrm{P}$-clone, and as a function of the number of clonal 536 generations since hatching of the the diapausing egg. Error bars show \pm 1 S.E. 


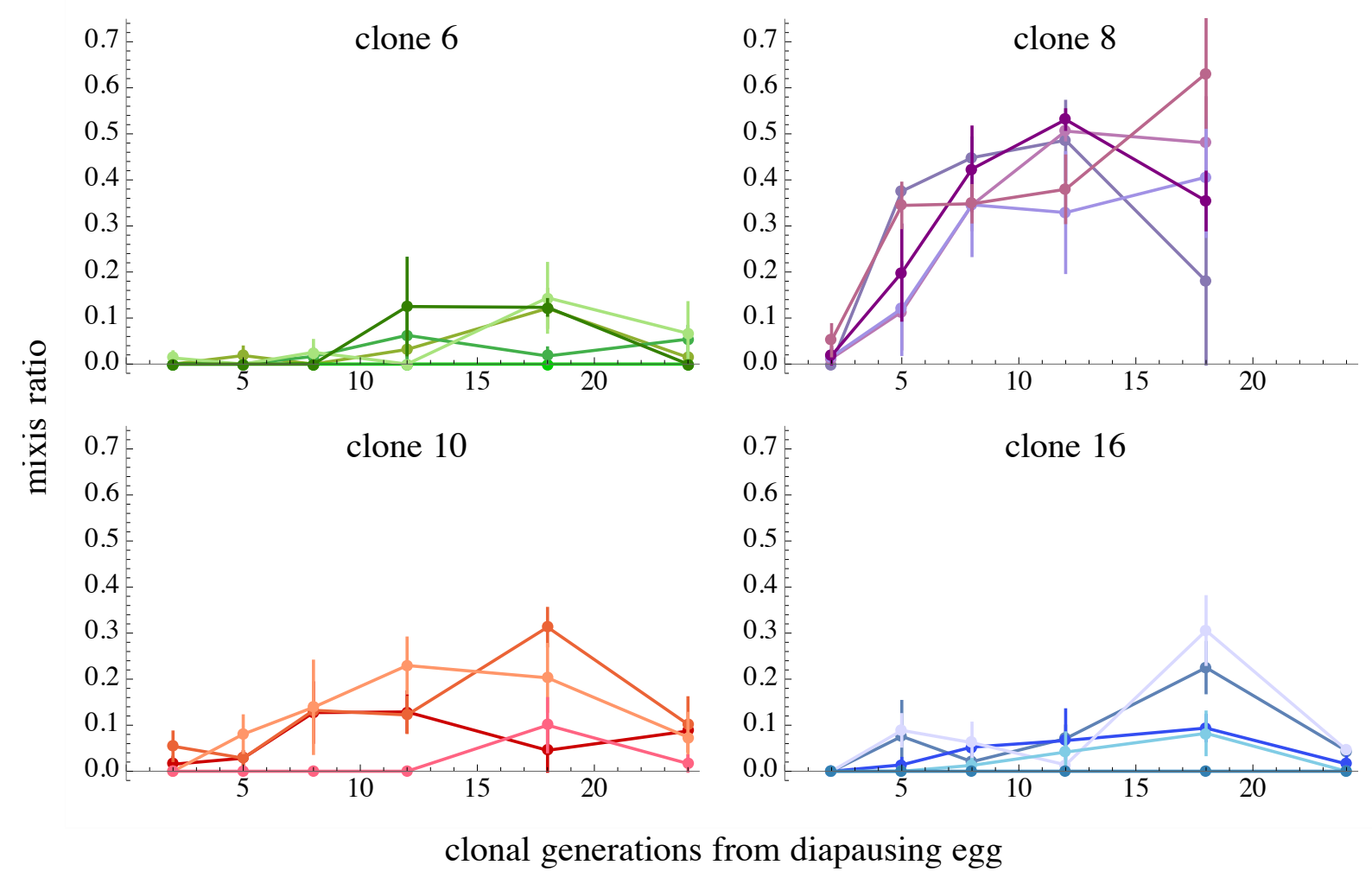

537

Figure 4. Mixis ratio (proportion of sexual females among the whole progeny of an individual) as a function of the number of clonal generations since hatching of the diapausing egg, averaged over each F1-clone (initiated from a different diapausing egg) ${ }_{541}$ produced by intraclonal mating within each P-clone ( 5 F1-clones from clones 6,8 and ${ }_{542} 16$, and 4 F1-clones for clone 10). Error bars show \pm 1 S.E. 


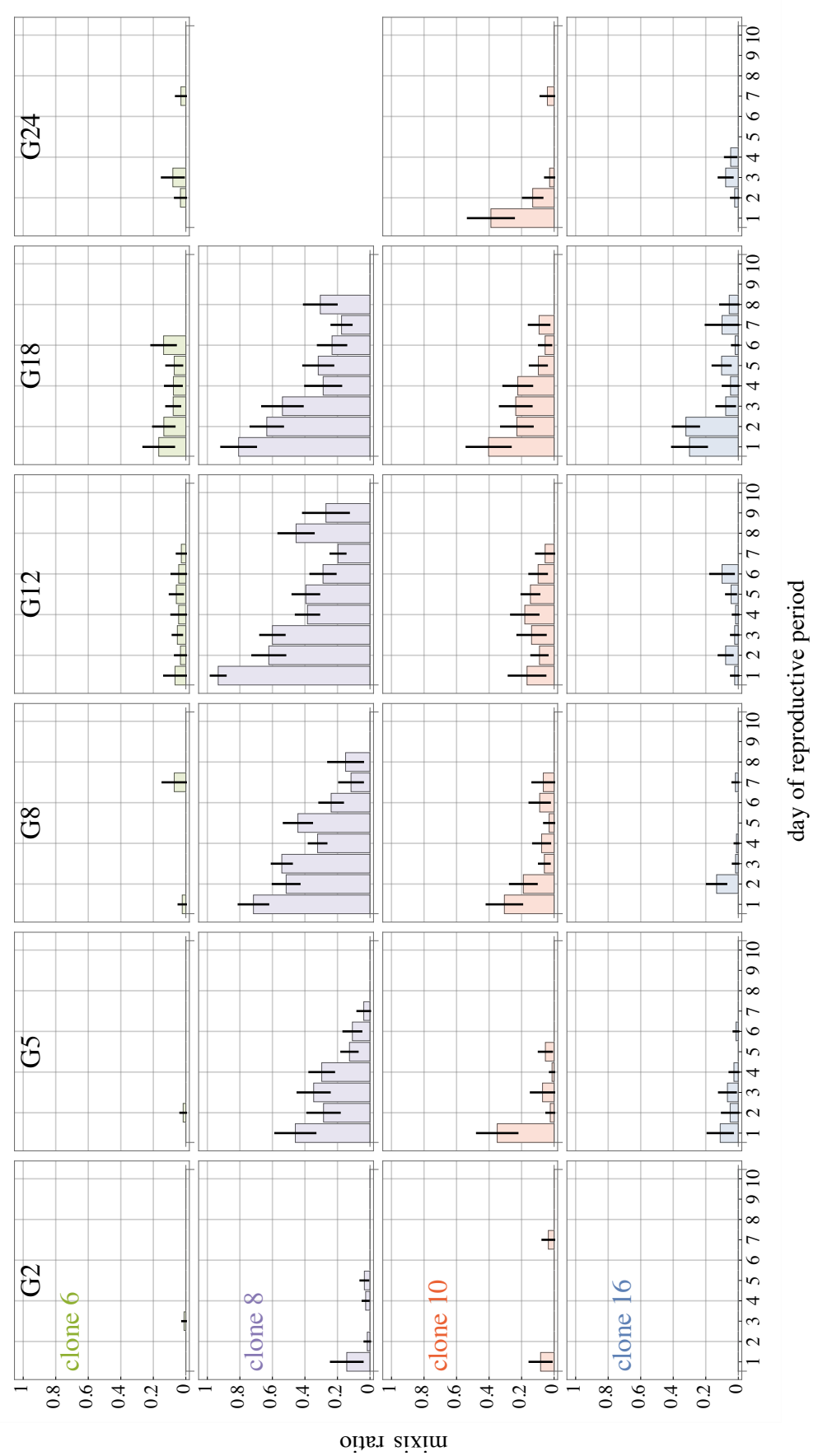

Figure 5. Mixis ratio (measured as the proportion of sexual females among offspring produced per day) as a function of the day of the reproductive period (day 1 corresponding to the day of first reproduction), for the different P-clones and numbers of clonal generations after hatching of the diapausing egg. Error bars show \pm 1 S.E. Averages were computed only when more than 10 juveniles were produced for a given mother's age class, over the whole P-clone and for a given generation. 